\title{
Extrapolation Methods for the Dirac Inverter in Hybrid Monte Carlo*
}

\author{
R. C. Brower, A.R. Levi ${ }^{\mathrm{a}}$ and K. Orginos ${ }^{\mathrm{b}}$ \\ a Department of Physics, Boston University, 590 Commonwealth Ave., Boston, MA 02215, USA \\ bepartment of Physics, Brown University, Providence, RI 02912, USA
}

In Hybrid Monte Carlo(HMC) simulations for full QCD, the gauge fields evolve smoothly as a function of Molecular Dynamics (MD) time. Thus we investigate improved methods of estimating the trial solutions to the Dirac propagator as superpositions of the solutions in the recent past. So far our best extrapolation method reduces the number of Conjugate Gradient iterations per unit MD time by about a factor of 4 . Further improvements should be forthcoming as we further exploit the information of past trajectories.

\section{INTRODUCTION}

The inclusion of internal fermion loops in the vacuum of $\mathrm{QCD}$ is a major challenge. The present state of the art for generating full QCD configurations is the so called Hybrid Monte Carlo algorithm which uses Molecular Dynamic evolution in a "fifth time" coordinate t. The Hamiltonian for this evolution is

$$
S=\frac{1}{2} \operatorname{Tr} P^{2}+S_{g}(U)+\varphi^{\dagger}\left[M^{\dagger} M\right]^{-1} \varphi
$$

where $P_{\mu}(x)$ are the angular momenta conjugate to the gauge fields $U_{\mu}(x), S_{g}(U)$ is the pure gauge action, $M(U)$ is the Dirac matrix and $\varphi$ is the pseudofermion field. In our discussion the precise form of the gauge action is not important. What is relevant is the need to accurately integrate the equations of motion, calculating the force on $U$ due to the pseudofermions at each time step. This requires solving, over and over again, the linear equation,

$$
A(t) \chi(t)=\varphi,
$$

where $A(t) \equiv M(U)^{\dagger} M(U)$ and $\chi(t)$ is the solution of the inverted Dirac operator. Technically this is achieved by starting with a trial value $\chi_{\text {trial }}$ and iteratively solving Eq. 2 for $\chi(t)$. On the order of 100 MD steps are taken holding the pseudofermion source fix, so that the operator $\mathrm{A}(\mathrm{t})$ changes smoothly as a function of the MD time

\footnotetext{
*Talk presented by A.R. Levi
}

$\mathrm{t}$, as new values of $\mathrm{U}$ are generated. These iterations, usually done by the conjugate gradient (CG) method, are the most computational expensive part of Hybrid Monte Carlo algorithms.

This raises an obvious question. As we move in MD time, why are we not able to "learn" from the recent past enough about the space of likely solutions to vastly improve our iterative scheme?

One should be able to give a very good estimate, $\chi_{\text {trial }}$ before starting the conjugate gradient routine. A crucial ingredient in this approach, is the fact that detail balance is in principle preserved independent of the starting trial configuration so long as one converges accurately to the solution. Therefore, we propose to estimate carefully the starting configuration[2]. To accomplish this some information on the configurations in the previous MD steps have to be stored. Although these algorithms will use more memory, memory is often not a severe constraint in modern super computer simulations.

\section{ANALYTIC EXTRAPOLATIONS}

To motivate our extrapolation methods, consider the function $\chi(t)$ as an analytic function of t. For simplicity of notation suppose we want the value at $\mathrm{t}=0$, given past values at $t_{1}, t_{2}, t_{3}, \cdots$. In practice this is usually a regular series of values $t_{i}=-i \epsilon$ with a integrations step of size $d t=\epsilon$. Then a trial value for the the solution, $\chi(0)$ of the new Dirac matrix $A(0)$, might be considered as a 
linear superposition of old solutions,

$\chi_{\text {trial }}=c_{1} \chi\left(t_{1}\right)+c_{2} \chi\left(t_{2}\right)+\cdots+c_{N} \chi\left(t_{N}\right)$.

If $N \epsilon$ is sufficiently small, we may Taylor expand each term around $\mathrm{t}=0$ and determine the coefficients by canceling all terms for $\epsilon^{k}$ to $O\left(\epsilon^{N}\right)$.

$\sum_{i=1}^{N}\left(t_{i}\right)^{n-1} c_{i}=\delta_{1, n}$

As we will demonstrate this procedure is equivalent to the familiar N-1 order polynomial fit to $\mathrm{N}$ points.

\subsection{Polynomial Extrapolation}

Good result can be obtained even with a si mple polynomial fit of degree $N-1$. To estimate the configuration it is necessary to store in memory the previous $(N+1)$ configurations. However the polynomial extrapolation does not require significant computational effort, it is just a local sum on each lattice point with fixed coefficient, that is less than a single CG step. The $\chi_{\text {trial }}$ is expressed as a polynomial, $\chi_{\text {trial }}=y_{1}+t y_{2}+$ $\cdots+t^{N-1} y_{N}$, whose coefficient satisfy the constraint,

$\sum_{n=1}^{N}\left(t_{i}\right)^{n-1} y_{n}=\chi\left(t_{i}\right)$.

One can easily prove that Eq. 4 and Eq. 5 define identical extrapolation $\left(y_{1}=\sum_{i} c_{i} \chi\left(t_{i}\right)=\chi_{\text {trial }}\right.$. For equally spaced time steps $t_{i}=-i \epsilon$, the coefficients given by

$c_{i}=(-1)^{i-1} \frac{N !}{i !(N-i) !}$.

Table 1 shows the results of simulations for polynomial extrapolation. The number of CG steps needed to reach convergence is plotted in function of the degree of extrapolation $N$, and the MD time step $\epsilon$. The case $N=1$ corresponds to starting with the old solution.

\subsection{Minimum Residual Extrapolation}

An alternate, perhaps more appealing, approach is to consider the past history of solutions, $\chi\left(t_{i}\right)$ as defining an important linear subspace for seeking an optimal trial solution. Since the Conjugate Gradient method is in fact just a minimal residual technique confined to the Krylov subspace spanned by vectors $A^{j-1} \chi_{\text {trial }}$, why not start by looking at a "smarter" subspace based on past success for nearby times?

In this spirit, we suggest minimizing the norm of the residual,

$r^{\dagger} r=\chi^{\dagger} M^{\dagger} M \chi-\varphi^{\dagger} \chi-\chi^{\dagger} \varphi+b^{\dagger} b$,

in the subspace spanned by $\chi_{i} \equiv \chi\left(t_{i}\right)$, where $r=b-M \chi$ and $b \equiv M^{\dagger} \varphi$. The minimization condition reduces to

$\sum_{j=1}^{N} \chi_{i}^{\dagger} M^{\dagger} M \chi_{j} c_{j}=\chi_{i}{ }^{\dagger} \varphi$.

The only problem is that this system can be ill conditioned because the past solutions $\chi_{i}$ differ from each other by order $\epsilon$. Nevertheless if we only want to get the minimum of $r^{\dagger} r$ in $\operatorname{span}\left(\chi_{i}\right)$ using a Gram Schmitt orthonormalization, we can solve the system avoiding the singularities.

This method requires $\left(N^{2}+5 N\right) / 2$ dots product and $(N) M \chi$ matrix-vector applications, and the storage of $(N)$ past configuration. It is interesting to note that this method gives coefficients that for the first few order reproduce very close to the polynomial extrapolation. Table 2 show the number of CG steps using this method.

\section{CONCLUSIONS}

To compare efficiencies, the CG iterations should be divided by $\epsilon$, so we compare the total number of CG steps needed to evolve the system for a fixed distance in configuration space. Note that if $\epsilon$ is too large, the overall performance is good, but the acceptance will drop drastically. If $\epsilon$ is too small, the extrapolation is excellent, but the system will evolve too slowly in phase space. It is not trivial that we find a window in $\epsilon$ where both the acceptance is good and the extrapolation works well. Moreover this window has parameters close to those used in actual simulations.

Our present approach is clearly not the only one worth considering. In fact we have emphasized the analytic properties of $\chi(t)$ because it suggests ways to understand and further improve 
Table 1

CG steps using polynomial extrapolation

\begin{tabular}{llllllll}
\hline $\mathrm{N}=1$ (previous data) & 0.98 & 0.93 & 0.93 & 0.88 & 0.85 & 0.77 & 0.63 \\
$\mathrm{~N}=2$ (1 $1^{\text {st }}$ order extrap.) & 0.92 & 1.07 & 0.86 & 0.74 & 0.72 & 0.62 & 0.27 \\
$\mathrm{~N}=3$ (2 $2^{\text {th }}$ order extrap.) & 0.91 & 0.82 & 0.74 & 0.61 & 0.59 & 0.47 & 0.21 \\
$\mathrm{~N}=4\left(3^{\text {th }}\right.$ order extrap.) & 0.96 & 0.77 & 0.56 & 0.44 & 0.41 & 0.31 & 0.21 \\
$\mathrm{~N}=5$ (4 $4^{\text {th }}$ order extrap.) & 0.86 & 0.77 & 0.54 & 0.45 & 0.42 & 0.35 & 0.26 \\
$\mathrm{~N}=6$ (5 $5^{\text {th }}$ order extrap.) & 0.70 & 0.48 & 0.39 & 0.38 & 0.40 & 0.40 & 0.41 \\
$\mathrm{~N}=7\left(6^{\text {th }}\right.$ order extrap. $)$ & 0.70 & 0.59 & 0.40 & 0.42 & 0.42 & 0.39 & 0.46 \\
\hline$\delta t=10^{-3} *$ & 15 & 10 & 9 & 8 & 7 & 5 & 2
\end{tabular}

Number of CG steeps to reach the solution (residue $<10^{-12}$ ), normalized to 1 for $\chi=0$. Full QCD configurations on $16^{4}$ lattice with Wilson fermions $k=0.157$ and $\beta=5.6$. Average on 30 configurations, statistical errors are of the order of $6 \%$.

Table 2

CG steps using minimum residual extrapolation

\begin{tabular}{llllllll}
\hline $\mathrm{N}=1$ & 0.99 & 0.93 & 0.92 & 0.87 & 0.84 & 0.81 & 0.64 \\
$\mathrm{~N}=2$ & 0.87 & 0.72 & 0.71 & 0.69 & 0.63 & 0.49 & 0.25 \\
$\mathrm{~N}=3$ & 0.85 & 0.64 & 0.57 & 0.52 & 0.49 & 0.32 & 0.06 \\
$\mathrm{~N}=4$ & 0.72 & 0.49 & 0.41 & 0.34 & 0.28 & 0.16 & 0.10 \\
$\mathrm{~N}=5$ & 0.72 & 0.37 & 0.31 & 0.23 & 0.19 & 0.12 & 0.07 \\
$\mathrm{~N}=6$ & 0.51 & 0.28 & 0.26 & 0.21 & 0.16 & 0.13 & 0.06 \\
$\mathrm{~N}=7$ & 0.49 & 0.25 & 0.24 & 0.21 & 0.19 & 0.12 & 0.06 \\
$\mathrm{~N}=8$ & 0.40 & 0.26 & 0.23 & 0.18 & 0.15 & 0.09 & 0.06 \\
$\mathrm{~N}=9$ & 0.38 & 0.23 & 0.19 & 0.16 & 0.13 & 0.09 & 0.06 \\
$\mathrm{~N}=10$ & 0.39 & 0.22 & 0.19 & 0.14 & 0.12 & 0.10 & 0.04 \\
$\mathrm{~N}=11$ & 0.38 & 0.18 & 0.17 & 0.14 & 0.11 & 0.08 & 0.04 \\
\hline$\delta t=10^{-3 *}$ & 15 & 10 & 9 & 8 & 7 & 5 & 2
\end{tabular}

Number of CG steeps to reach the solution (residue $<10^{-12}$ ), normalized to 1 for $\chi=0$. Same configurations of Table 1. Statistical errors are of the order of $4 \%$.

our approach. For example the failure at fixed $\epsilon$ to improve the polynomial extrapolation by increasing indefinitely the number of terms is probably a signal of nearby singularities in t. Our success so far is probably due to low eigenvalues of the Dirac operator changing more slowly with time.

Many other ways of using the past to avoid needless repetition can be imagined[3]. For example the CG routine itself generates vectors, that may be useful than solutions in the more distant past. A Karman filter can be used top introduce exponential decreasing information from the past without increasing the storage requirement. The subspace of past vectors might be used not just as a way to arrive at an initial guess, but also as a way to precondition the iterative process it- self. For now, we are pleased for now that even a few vectors from the past can be combined with simple extrapolation ideas to give a very useful acceleration method.

\section{REFERENCES}

1. S. Gottlieb, W. Liu, D. Toussaint, R.L. Renken and R.L. Sugar, Phys Rev. D 35 (1987) 2531.

2. R. Brower, A.R. Levi and K. Orginos, in preparation.

3. "Those who cannot remember the past are condemned to repeat it.", George Santayana, The Life of Reason or Phases of Human Progress, New York, 1917. 